\title{
Inferência bayesiana aplicada à estimação de herdabilidades dos parâmetros da curva de crescimento de fêmeas da raça Nelore
}

\author{
Bayesian inference applied to the estimation of heritability of growth curve parameters of \\ Nellore females
}

\author{
Analía del Valle Garnero ${ }^{\mathrm{I}}$ Cintia Righetti Marcondes ${ }^{\mathrm{II}}$ Ronyere Olegário de Araújo ${ }^{\mathrm{III}}$ \\ Henrique Nunes de Oliveira ${ }^{\mathrm{IV}}$ Raysildo Barbosa Lôbo ${ }^{\mathrm{v}}$
}

RESUMO

Objetivou-se estimar parâmetros genéticos, utilizando inferência Bayesiana, para as estimativas dos parâmetros individuais de peso à maturidade ( $\hat{\mathrm{A}})$ e taxa de crescimento ( $\hat{\mathrm{K}})$, obtidos pela função de crescimento Brody. $O$ arquivo estava constituído de 14.563 registros de pesos e idades referentes a 1.158 fêmeas da raça Nelore, participantes do Programa de Melhoramento Genético da Raça Nelore. Para a análise das estimativas dos parâmetros da curva, via inferência bayesiana, foi proposto um modelo animal unicaráter, que incluiu como fixo o efeito de grupo contemporâneo (animais nascidos no mesmo estado, no mesmo trimestre do ano, mesmo ano e mesmo regime alimentar) e como aleatórios os efeitos genético direto e residual. Nessa análise, foram utilizados dois diferentes tamanhos para as cadeias geradas pelo algoritmo de amostragem de Gibbs, de 550 e 1.100 mil ciclos, com períodos de descarte amostral de 50 e 100 mil ciclos, respectivamente, e amostragens a cada 500 e 1.000 ciclos, respectivamente. As médias posteriores da variância genética aditiva e residual foram próximas, tanto para $\hat{\mathrm{A}}$ quanto para a $\hat{\mathrm{K}}$, mesmo quando implementados diferentes tamanhos para as cadeias geradas pelo algoritmo de amostragem de Gibbs. Os coeficientes de herdabilidade estimados para Â, variaram de 0,44 a 0,46, amplitude semelhante aos 0,46 a 0,48 obtidos para as estimativas de $\hat{\mathrm{K}}$. Essas magnitudes indicam que a seleção pode ser usada como instrumento para alterar a forma da curva de crescimento desses animais. Entretanto, o uso das informações obtidas, visando à alteração da curva de crescimento dos animais, deve ser feito com grande cautela, uma vez que as características a serem trabalhadas na modificação do formato da curva de crescimento, de acordo com resultados da literatura especializada, são negativamente correlacionadas.

Palavras-chave: modelo animal, peso assintótico, raça Nelore, taxa de crescimento.

\section{ABSTRACT}

The objective of this study was to estimate genetic parameters using Bayesian inference for the estimates of individual parameters of mature weight $(\hat{\mathrm{A}})$ and growth rate $(\hat{\mathrm{K}})$, obtained by Brody growth function. The file consisted of 14,563 records relating to weights and ages of 1,158 Nelore females, participants in the Genetic Improvement Program of the Nellore. For the estimates analysis of the curve parameters via Bayesian inference, it was used an univariated animal model that included as fixed effect of contemporary group (animals born on the same state, in the same quarter of the year, the same year and feedlot) and random as the direct genetic and residual. In this analysis it was used two different sizes for the chains generated by Gibbs sampling algorithm, 550 and 1.1 million cycles, with initial discarding of 50000 and sample of 100000 cycles, respectively, and sampled every 500 cycles and 1000, respectively. The mean posterior and residual additive genetic variance showed a small variation to both $\hat{\mathrm{A}}$ and $\hat{\mathrm{K}}$, even when implemented in different sizes for the chains generated by Gibbs sampling algorithm. The heritability estimates for $\hat{\mathrm{A}}$, ranged from 0.44 to 0.46 , similar to the range 0.46 to 0.48 obtained for the estimates of $\hat{\mathrm{K}}$. These magnitudes indicate that the selection can be used as a tool to change the shape of the growth curve of these animals. However, the use of the information to amend the growth curve of animals, must be done with great care, since the traits to be worked in the modification of the shape of the growth curve according to the literature results are negatively correlated.

Key words: animal model, asymptotic weight, growth rate, Nellore breed.

${ }^{I}$ Universidade Federal do Pampa (UNIPAMPA), Campus de São Gabriel, 97300-000, São Gabriel, RS, Brasil. E-mail: analiagarnero@yahoo.com.br. Autor para Correspondência.

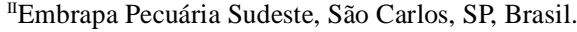

"IIPrograma de Pós-graduação em Ciências Animais, Faculdade de Agronomia e Medicina Veterinária (FAV), Universidade de Brasília (UnB), Brasília, DF, Brasil.

${ }^{\text {IV }}$ Departamento de Zootecnia, Faculdade de Ciências Agrárias e Veterinárias (FCAV), UNESP, Jaboticabal, SP, Brasil.

vAssociação Nacional de Criadores e Pesquisadores (ANCP), Ribeirão Preto, SP, Brasil. 


\section{INTRODUÇÃO}

Análises de dados de medidas repetidas se mostram de grande importância no melhoramento genético, pois incluem as situações em que as unidades experimentais ou indivíduos, de diferentes subpopulações ou tratamentos (sexo, raça, etc.), são analisados ao longo de diversas condições de avaliação (tempo, doses etc). Entre essas análises, destacam-se as curvas de crescimento dos animais que relacionam os pesos $(y)$ e as idades $(t)$, por meio de modelos não-lineares (Logístico, Gompertz, Brody, Von Bertalanffy, Richards) que apresentam parâmetros com interpretação biológica (FITZHUGH JR., 1976). Esses modelos são utilizados para descrever o crescimento do animal ao longo do tempo, o que possibilita avaliar os fatores genéticos e de ambiente que influenciam na forma da curva de crescimento e, desse modo, alterá-la por meio da identificação e seleção de animais com maior velocidade de crescimento, sem alterar o peso adulto, ao invés de selecionar animais cada vez maiores, aumentando os custos de mantença (GARNERO et al., 2006). GARNERO et al. (2005) e SANTORO et al. (2005) destacaram o modelo não linear de Brody como adequado para a descrição do crescimento de bovinos da raça Nelore, principalmente por apresentar formulação matemática relativamente simples.

Coeficientes de herdabilidade para as estimativas dos parâmetros das curvas, obtidos por meio de ajustes de modelos de crescimento, geralmente são restritos aos parâmetros de maior significado biológico, como o peso assintótico $(A)$ e a taxa de maturidade $(K)$. Assim, herdabilidades estimadas para $A$ na raça Nelore tiveram ampla variação, de 0,09 a 0,69 (GARNERO et al., 2005; SILVA et al., 2010). Para $K$, as estimativas também foram de grande variação e de moderada a alta magnitude, não ultrapassando o valor de 0,44 (SANTORO et al., 2005; SILVA et al., 2010).

Uma metodologia utilizada para a obtenção de parâmetros genéticos para as estimativas dos parâmetros das curvas, bem como para a avaliação genética, baseia-se na teoria Bayesiana. A inferência Bayesiana utiliza métodos probabilísticos para descrever a incerteza sobre o verdadeiro valor de algum parâmetro (FARIA et al., 2007), ou seja, consiste em descrever erros que possam existir em torno da estimativa de um parâmetro, usando como medida do erro a probabilidade de que o parâmetro apresente determinado valor.
Assim, objetivou-se estimar os componentes de variância e as herdabilidades, utilizando inferência Bayesiana, a partir das estimativas dos parâmetros individuais de crescimento $\hat{\mathrm{A}}$ e $\hat{\mathrm{K}}$, obtidos pelo modelo clássico não linear Brody, com dados de crescimento até a maturidade de fêmeas da raça Nelore.

\section{MATERIAL E MÉTODOS}

Os 14.563 registros de pesos e idades referentes a 1.158 fêmeas da raça Nelore, nascidas entre 1985 e 1995, foram provenientes de dez fazendas participantes do Programa de Melhoramento Genético da Raça Nelore (PMGRN) e distribuídas em sete Estados do Brasil (BA, GO, MG, MS, PA, PR e $\mathrm{SP})$. A função Brody, $\mathrm{Y}_{\mathrm{t}}=A\left(1-\mathrm{B} e^{-k t}\right)+\varepsilon$ (BRODY, 1945), foi o modelo matemático não linear utilizado para estimar os parâmetros da curva de crescimento, uma vez que há indícios na literatura do melhor ajuste dos dados da raça Nelore a esse modelo (GARNERO et al., 2005; MALHADO et al., 2009; SILVA et al., 2011). Nessa equação, $Y$ representa o peso do animal a uma determinada idade (t); Â é o valor assintótico de $Y t$ (peso médio na maturidade, em $\mathrm{kg}$ ); $B$ é a constante de integração relacionada com os pesos iniciais (grau de maturidade do animal ao nascimento); $\hat{\mathrm{K}}$ é a taxa de variação da função exponencial (velocidade, em $\mathrm{kg} \mathrm{dia}{ }^{-1}$, com que o animal se aproxima ao tamanho adulto); $e$ é o logaritmo em base natural e e representa o erro aleatório, associado à cada pesagem, no qual foi assumido que os erros são independentes e normalmente distribuídos com média zero e variância igual a $\sigma_{\theta}^{2}$, ou seja, $e_{i j} \sim N\left(0 ; \sigma_{\theta}^{2}\right)$. Foi utilizado o procedimento NLIN do SAS (2003) para a obtenção das estimativas individuais dos parâmetros e dos parâmetros médios, a partir da totalidade dos dados disponíveis. Vale ressaltar que ocorreu a convergência de todos os modelos dos animais utilizados no presente estudo.

Para a obtenção dos componentes de variância e herdabilidades das estimativas dos parâmetros pesos assintóticos ( $\hat{A})$ e taxas de maturidade ( $\hat{\mathrm{K}})$, utilizou-se o programa Multiple Trait Gibbs Sampling in Animal Model (VAN TASSEL \& VAN VLECK, 1996), sob modelo animal. Nessa análise, foi proposto um modelo unicaráter, que incluiu como fixo o efeito de grupo contemporâneo (GC) e, como aleatórios, os efeitos genético aditivo direto e residual. Os GC foram constituídos por animais nascidos no mesmo estado, no mesmo 
trimestre do ano (Estação $1=$ meses de janeiro a março, $2=$ meses de abril a junho, $3=$ meses de julho a setembro, $4=$ mês outubro a dezembro), mesmo ano e mesmo regime alimentar $(1=$ pasto, $2=$ semiconfinado, 3 = confinado). Foram eliminados animais que não possuíam pelo menos uma pesagem entre os 24 e 36 meses, uma entre 36 e 48 meses e uma após os 54 meses. Também foram eliminados todos os GC com menos de cinco animais, permanecendo 153 grupos e 1087 registros para as análises. A matriz de parentesco continha 1900 animais.

Em termos matriciais, o modelo animal pode ser descrito como: $\mathrm{y}=\mathrm{X} \beta+\mathrm{Za}+\varepsilon$, em que: $\mathrm{y}=$ vetor das observações de cada característica (parâmetro $\hat{\mathrm{A}}$ e $\hat{\mathrm{K}}$ ); $\mathrm{X}=$ matriz de incidência dos efeitos fixos (GC); $\beta=$ vetor dos efeitos fixos; $Z=$ matriz de incidência do efeito genético direto de cada animal; $\mathrm{a}=$ vetor de efeitos genéticos diretos aleatórios; $\varepsilon=$ vetor de efeitos residuais aleatórios.

As pressuposições acerca da distribuição de $y, a$, e $\varepsilon$ podem ser descritas como:

$$
\left[\begin{array}{l}
y \\
a \\
\varepsilon
\end{array}\right] \approx N M V\left\{\left[\begin{array}{l}
X \beta \\
0 \\
0
\end{array}\right]\left[\begin{array}{ccc}
V & Z G & R \\
G Z^{\prime} & G & 0 \\
R & 0 & R
\end{array}\right]\right\}
$$

em que: $V=Z G Z+R ; G=A \sigma_{a}^{2} ; R=I \sigma_{e}^{2}$.

Para os componentes de (co)variância genética, foi assumido que $G$ têm distribuição de Wishart Invertida $(I W)$, na forma: $G \sim I W\left(G_{0}, v_{g}\right)$

Por outro lado, para os componentes de variância residual, foi assumido que cada elemento $\sigma_{\theta}^{2}$ tem distribuição qui-quadrado invertida, na forma: $\sigma_{\varepsilon}^{2} \sim I X^{2}\left(S_{0}, v_{r}\right)$

A função densidade de probabilidade conjunta posterior dos parâmetros, dados os hiperparâmetros, foi obtida por meio do produto das distribuições a priori pela função de verossimilhança, conforme descrito por VAN TASSEL \& VAN VLECK (1996). Para implementar a amostragem de Gibbs, foram obtidas as funções densidade de probabilidade condicionais completas para cada parâmetro, por meio da derivação da função de densidade posterior conjunta, considerando como constantes os valores dos outros parâmetros contidos no modelo. As estimativas das médias a posteriori para os componentes de (co) variância foram baseadas no valor esperado de suas distribuições condicionais completas.

O programa Gibanal (VAN KAAM, 1997) foi empregado para análise das cadeias Gibbs, com o objetivo de definir o período de burn-in, intervalo de amostragem do parâmetro da cadeia e número total de amostras a ser empregado. Com isso, foram gerados dois diferentes tamanhos para a cadeia de Markov, de 550.000 e 1.100 .000 ciclos. Os períodos de descarte amostral (burn in) foram de 50.000 e 100.000 ciclos, respectivamente. As amostras foram realizadas (thinning interval) a cada 500 e 1.000 ciclos respectivamente, assegurando que as correlações seriais fossem nulas ou muito pequenas, segundo VAN TASSEL \& VAN VLECK (1996). Neste estudo, os valores iniciais para cada componente de variância de $A$ e $K$ foram resultantes de análises prévias (GARNERO et al., 2005), a partir do Multiple Trait Derivative Free Restricted Maximum Likelihood- MTDFREML (BOLDMAN et al., 1995). A distribuição inicial dos parâmetros foi assumida como sendo flat, não refletindo nenhum conhecimento inicial do parâmetro estudado (0), e aguda, admitindo como distribuição informativa para os valores dos parâmetros (3). O critério de convergência usado na interação Gauss-Seidel foi de $10^{-9}$. $\mathrm{O}$ arquivo gerado pelo MTGSAM foi submetido a análises, a posteriori, pelo programa GIBANAL (VAN KAAM, 1997), para a obtenção das densidades marginais posteriores das herdabilidades das estimativas do $\hat{\mathrm{A}}$ e de $\hat{\mathrm{K}}$.

\section{RESULTADOS E DISCUSSÃO}

As médias das estimativas dos parâmetros peso assintótico $(\hat{\mathrm{A}})$ e da taxa de maturidade $(\hat{\mathrm{K}})$ foram $535,08 \mathrm{~kg}$ e $0,045 \mathrm{~kg} \mathrm{dia}^{-1}$, respectivamente, valores estes compatíveis aos $470 \mathrm{~kg}$ observados para Â em outros sistemas de produção (SANTORO et al., 2005; LOPES et al., 2011). Para $\hat{\mathrm{K}}$, notamse valores inferiores aos relatados para essa raça em outros estudos (MALHADO et al., 2009), caracterizando crescimentos mais tardios e peso assintótico superior para a presente população. Esses resultados eram esperados, visto ser clássica a relação antagônica entre as estimativas dos parâmetros $\hat{\mathrm{A}}$ e $\hat{\mathrm{K}}$ em diversos estudos envolvendo várias espécies (FREITAS, 2005; GARNERO et al., 2005), além de existir efeito de sexo tanto sobre o $\hat{A}$ quanto em relação a $\hat{\mathrm{K}}$. Contudo, ressalta-se a consistência das estimativas desses parâmetros, haja vista que a maioria dos trabalhos sobre esse tema não apresentam frequências de pesagens constantes e que representem valores observados do nascimento a fase adulta, considerando animais acima de quatro anos de idade (SANTORO et al., 2005; MALHADO et al., 2009; LOPES et al., 2011).

De modo geral, as médias posteriores da variância genética aditiva (Va) e residual (Vr) foram próximas (Tabela 1), mesmo quando implementados 
Tabela 1 - Estimativas da variância genética aditiva e residual e da herdabilidade $\left(\mathrm{h}^{2}\right)$, para as estimativas dos paràmetros $\hat{\mathrm{A}} \mathrm{e} \hat{\mathrm{K}}$, obtidas por inferência bavesiana.

\begin{tabular}{|c|c|c|c|c|c|c|c|c|c|c|c|c|c|c|}
\hline \multirow{2}{*}{$\mathrm{CM}^{*}$} & & \multirow{2}{*}{ hiper } & \multicolumn{6}{|c|}{ 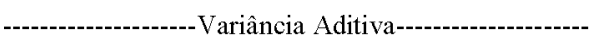 } & \multicolumn{5}{|c|}{ 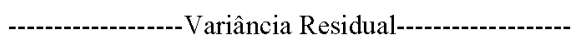 } & \multirow{2}{*}{$h^{2}$} \\
\hline & & & $\mathrm{CC}$ & $\sigma_{a}^{2}$ & Med & Mo & $\mathrm{IC}_{\mathrm{a}} 90 \%$ & $\mathrm{AD}_{\mathrm{a}}$ & $\sigma_{e}^{2}$ & Me & Mo & $\mathrm{IC}_{\mathrm{e}} 90 \%$ & $\mathrm{AD}_{\mathrm{e}}$ & \\
\hline \multirow{5}{*}{$\hat{\mathrm{A}}$} & \multirow{2}{*}{550} & 0 & 47 & 2982 & 2925 & 3397 & $\begin{array}{l}1556,5 \text { a } \\
4387,1\end{array}$ & $\begin{array}{l}2631 \mathrm{a} \\
3494\end{array}$ & 3486 & 3497 & 3373 & $\begin{array}{l}2505,1 \mathrm{a} \\
4750,5\end{array}$ & $\begin{array}{l}3245 a \\
4014\end{array}$ & 0,46 \\
\hline & & 3 & 50 & 2828 & 2739 & 2740 & $\begin{array}{l}1463,3 a \\
4445,6\end{array}$ & $\begin{array}{l}2658 \mathrm{a} \\
2953\end{array}$ & 3589 & 3622 & 3622 & $\begin{array}{l}2282,7 \text { a } \\
4837,2\end{array}$ & $\begin{array}{l}3581 \text { a } \\
4108\end{array}$ & 0,44 \\
\hline & & & & & & & & & & & & & & \\
\hline & \multirow{2}{*}{1.100} & 0 & 33 & 2983 & 2933 & 1957 & $\begin{array}{l}1581,8 \mathrm{a} \\
4470,7\end{array}$ & $\begin{array}{l}2301 \text { a } \\
3407\end{array}$ & 3485 & 3505 & 2438 & $\begin{array}{l}2254,3 \text { a } \\
4741,4\end{array}$ & $\begin{array}{l}3033 a \\
4032\end{array}$ & 0,46 \\
\hline & & 3 & 50 & 2889 & 2845 & 2845 & $\begin{array}{l}1483,5 \text { a } \\
4387,2\end{array}$ & $\begin{array}{l}2323 \mathrm{a} \\
3342\end{array}$ & 3539 & 3558 & 3558 & $\begin{array}{l}2283,5 \text { a } \\
4799,7\end{array}$ & $\begin{array}{l}3037 \mathrm{a} \\
4191\end{array}$ & 0,45 \\
\hline \multirow{5}{*}{$\hat{\mathrm{K}}^{1}$} & \multirow{2}{*}{550} & 0 & 37 & 61,3 & 60 & 63 & $\begin{array}{l}31,8 \mathrm{a} \\
93,1\end{array}$ & $\begin{array}{l}56,8 \mathrm{a} \\
68,4\end{array}$ & 65,9 & 66 & 54 & $\begin{array}{l}39,1 \text { a } \\
92,6\end{array}$ & $\begin{array}{l}60,2 a \\
81,1\end{array}$ & 0,48 \\
\hline & & 3 & 38 & 60,1 & 59 & 28 & $\begin{array}{l}31,9 \text { a } \\
92,8\end{array}$ & $\begin{array}{l}48,8 \text { a } \\
63,1\end{array}$ & 66,5 & 66 & 32 & $\begin{array}{l}39,9 \text { a } \\
91,6\end{array}$ & $\begin{array}{l}54,6 \mathrm{a} \\
79,5\end{array}$ & 0,47 \\
\hline & & & & & & & & & & & & & & \\
\hline & \multirow{2}{*}{1.100} & 0 & 37 & 61,5 & 66 & 40 & $\begin{array}{l}33,1 \text { a } \\
94,6\end{array}$ & $\begin{array}{l}46,2 \mathrm{a} \\
72,3\end{array}$ & 65,8 & 68 & 49 & $\begin{array}{l}38,4 \text { a } \\
91,8\end{array}$ & $\begin{array}{l}53,3 \mathrm{a} \\
79,4\end{array}$ & 0,48 \\
\hline & & 3 & 37 & 59,1 & 58 & 46 & $\begin{array}{l}29,4 \mathrm{a} \\
91,8\end{array}$ & $\begin{array}{l}44,7 \mathrm{a} \\
73,7\end{array}$ & 67,3 & 68 & 49 & $\begin{array}{l}40,2 \text { a } \\
92,8\end{array}$ & $\begin{array}{l}56,23 \text { a } \\
84,0\end{array}$ & 0,46 \\
\hline
\end{tabular}

*Cadeia de Markov em mil; hiper - hiperparàmetros das prioris; Critério de Convergência $(\mathrm{CC})$, variância genética $\left(\sigma_{a}^{2}\right)$, mediana (Med), moda (Mo), intervalo de credibilidade a $90 \%\left(\mathrm{IC}_{\mathrm{a}} 90 \%\right)$ para $\sigma_{a}^{2}$, alta-densidade a posteriori para $\sigma_{a}^{2}\left(\mathrm{AD}_{\mathrm{a}}\right)$.

${ }^{1}$ estimações $\times 10^{-3}$.

diferentes tamanhos para as cadeias de Markov, período de descarte (burn in) e tamanho amostral (thining interval). Da mesma forma, os intervalos de credibilidade a $90 \%$ e as regiões de alta densidade a posteriori apresentaram pequena amplitude de variação, quando comparados os resultados obtidos a partir das diferentes implementações. As distribuições posteriores para os componentes de Va e Vr (Tabela 1; Figura 1) para o $\hat{A}$, utilizando 550 mil ciclos e distribuições com hiper 0 e hiper 3 (hiperparâmetros da priori), apresentaram simetria, com região de alta densidade a posteriori entre 2631,08 e $3494,23 \mathrm{~kg}$ para a Va, e 3245,17 a 4108,66kg para a Vr (Tabela 1; Figura 1), valores estes superiores aos 1354,83 e aos $1431,07 \mathrm{~kg}$, estimados para a Va e Vr, respectivamente, por JENKINS et al. (1991). Chama-se a atenção para o trabalho destes últimos autores que utilizaram a metodologia dos quadrados mínimos para estimação dos componentes de variância. De forma semelhante, comportaram-se as distribuições posteriores para as Va e $\operatorname{Vr}$ (Tabela 1) quando se utilizaram 1.100 mil ciclos. A única diferença existente entre as distribuições foi no achatamento, quando se utilizaram distribuições a priori não informativa (flat), contudo, apresentaram regiões de alta densidade a posteriori coincidentes, localizadas entre $2.301,08$ e $3.407,88$ para a Va e 2.254,30 a 4.799,71 para a Vr.

As distribuições posteriores da $\mathrm{Va}$ e $\mathrm{Vr}$ para a taxa de maturação ( $\hat{\mathrm{K}}$ ), estimadas a partir da função Brody, comportaram-se de forma semelhante às do parâmetro Â (Figura 2). Quando utilizados 550 mil ciclos para a Va, observou-se simetria na distribuição posterior, com regiões de alta densidade entre 48,79 a 63,39 (Figura 2), não se verificando diferenças entre as distribuições iniciais, porém com leve achatamento da distribuição quando utilizada distribuição informativa igual a 3 .

Os coeficientes de herdabilidade estimados para Â variaram de 0,44 a 0,46 (Tabela 1), valores superiores aos 0,10 relatados por SANTORO et al. (2005) e aos 0,13 obtidos por MALHADO et al. (2009), trabalhos com animais da raça Nelore. Para o parâmetro $\hat{\mathrm{K}}$, a variação sumarizada para esse coeficiente no presente trabalho $(0,46$ a 0,48$)$ foi 


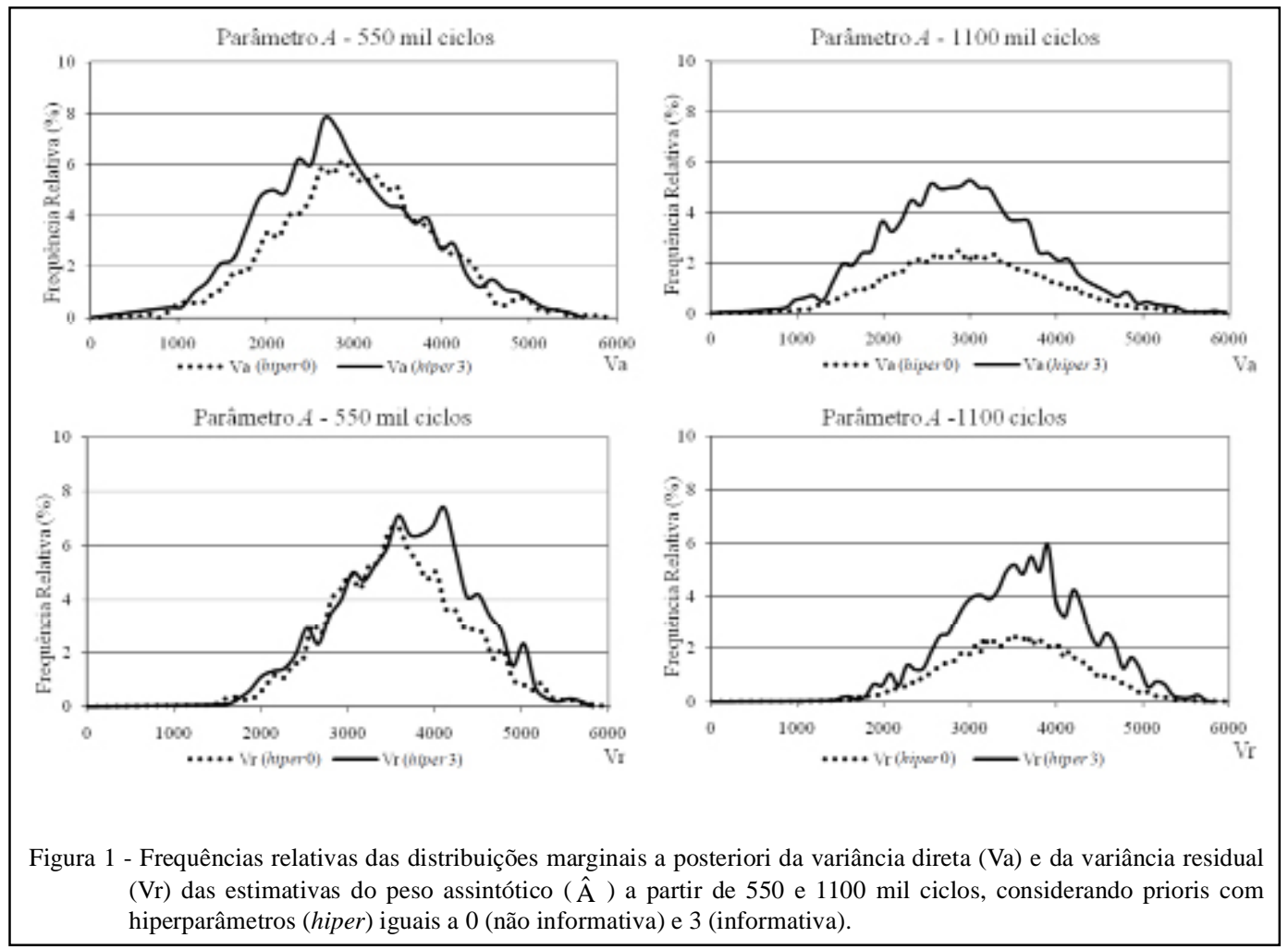

superior aos 0,22 estimados por SANTORO et al. (2005) e aos 0,14 obtidos por MALHADO et al. (2009), ambos trabalhos com animais da raça Nelore, utilizando a mesma função não linear adotada.

Os coeficientes de herdabilidade, tanto para $\hat{A}$ quanto para $\hat{\mathrm{K}}$, foram de elevada magnitude (Tabela 1), o que indica que a seleção poderia ser usada como instrumento para alterar a forma da curva de crescimento desses animais, como ressaltado por SILVA et al. (2010). Entretanto, o uso das informações obtidas nos rebanhos estudados em um programa de melhoramento genético, visando à alteração da curva de crescimento dos animais, deve ser feito com grande cautela, uma vez que as características a serem trabalhadas na modificação do formato da curva de crescimento (peso ao nascimento e taxa de maturação) seriam negativamente correlacionadas (FITZHUGH Jr., 1976), além também da grande variabilidade das estimativas. Nesse caso, os índices de seleção restritos poderiam ser utilizados com maior sucesso (DZAMA et al., 2001; SANTORO et al., 2005). BROWN et al. (1976) destacaram que a taxa de maturidade $(\hat{\mathrm{K}})$ poderia ser o único parâmetro disponível para o trabalho da indústria.
A partir dessa discussão, observamse variações nas estimativas de herdabilidade para os parâmetros da função de crescimento. Em princípio, isso se deve às diferenças verdadeiras no quociente variância aditiva/variância fenotípica entre populações para um mesmo caráter e espécie, além de diferenças na metodologia utilizada para estimação desses parâmetros, principalmente quando se utilizam, na inferência bayesiana, distribuições iniciais informativas. Porém, outras causas são inerentes à quantidade de dados analisados nestes trabalhos, uma vez que essas estimações em populações menores acarretam em erros-padrão mais expressivos em função ao valor pontual (metodologia frequentista), situação que é contornada pela aplicação da inferência bayesiana (FARIA et al., 2007).

\section{CONCLUSÃO}

As características relacionadas à curva de crescimento de fêmeas da raça Nelore são passíveis de seleção, dados os valores estimados dos parâmetros genéticos por meio da inferência bayesiana. Se aplicadas aos programas de melhoramento como critérios de seleção, não favorecerão o aumento do peso adulto das fêmeas na raça estudada. 

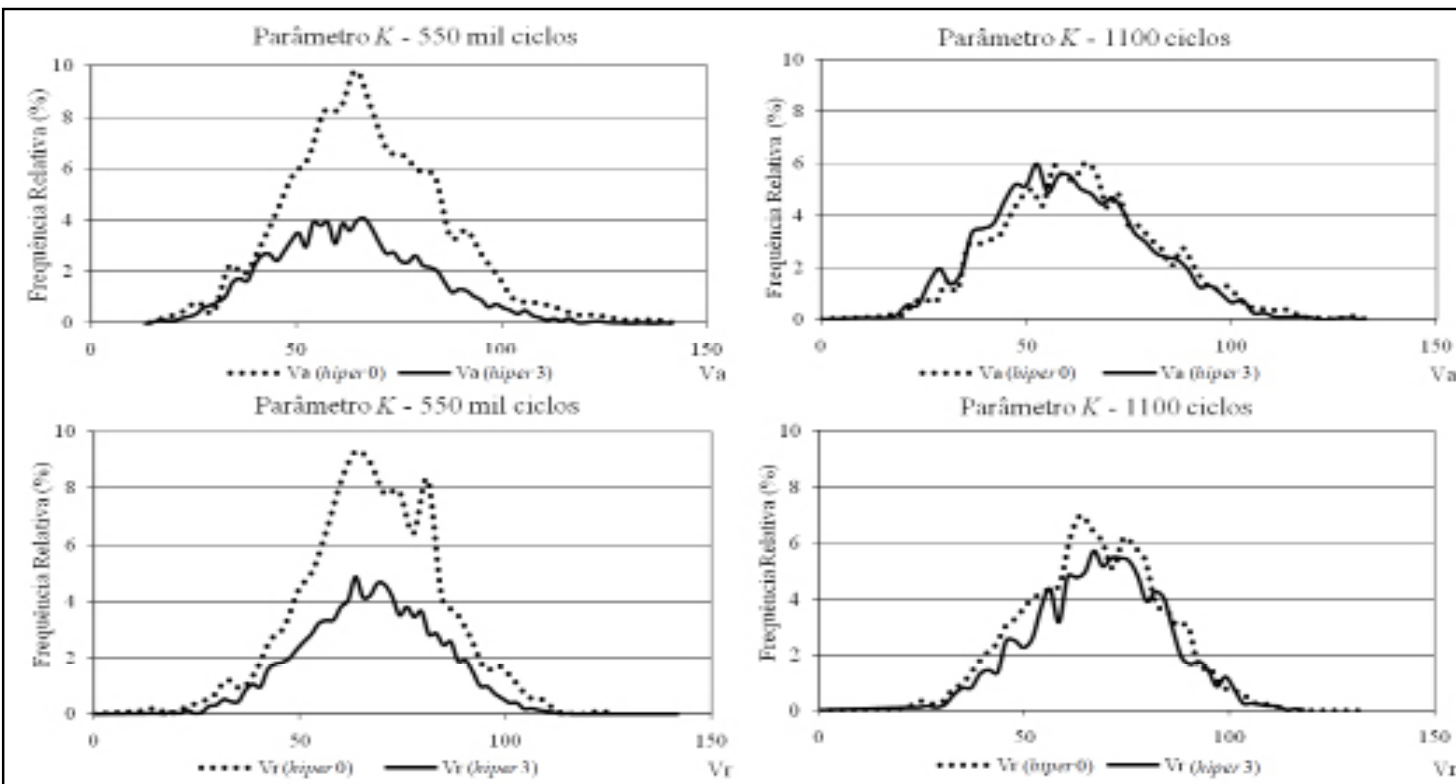

Figura 2 - Distribuições marginais a posteriori da variância direta (Va) e da variância residual (Vr) das estimativas da taxa de maturidade $(\hat{\mathbf{K}})$ a partir de 550 e 1100 mil ciclos, considerando prioris com hiperparâmetros (hiper) iguais a 0 (não informativa) e 3 (informativa).

\section{REFERÊNCIAS}

BOLDMAN, K.G.et al. A manual for use of MTDFREML. A set of program to obtain estimates of variances and covariances (DRAFT). Lincoln: Departament of Agriculture/Agricultural Research Servica, 1995. 120p.

BRODY, S. Bioenergetics and growth. New York: Rheinhold, 1945. 645p

BROWN, J.E. et al. A comparison of nonlinear models for describing weight-age relationships in cattle. Journal of Animal Science, v.42, p.810-818, 1976. Disponível em: <http://jas.fass. org/content/42/4/810>. Acesso em: 29 fev. 2012

DZAMA, K. et al. Index selection of beef cattle for growth and milk production using computer simulation modelling. South African Journal of Animal Science, v.31, p.65-75, 2001. Disponível em: <http://www.ajol.info/index.php/sajas/article/ viewFile/3832/11770>. Acesso em: 29 fev. 2012.

FARIA, C.U. et al. Inferência Bayesiana e sua aplicação na avaliação genética de bovinos da raça Nelore: revisão bibliográfica. Ciência Animal Brasileira, v.8, n.1, p.75-86, 2007. Disponível em: <http://www.revistas.ufg.br/index.php/vet/article/ view/1161/1251>. Acesso em: 29 fev. 2012.

FITZHUGH Jr., H.A. Analysis of growth curves and strategies for altering their shape. Journal of animal Science, v.42, p.1036-1051, 1976. Disponível em: <http://jas.fass.org/content/42/4/1036>. Acesso em: 29 fev. 2012.

FREITAS, A.R. Curvas de crescimento na produção animal. Revista Brasileira de Zootecnia, v.34, p.786-795, 2005.
Disponível em: <http://www.scielo.br/scielo.php?script=sci_artte xt\&pid=S1516-35982005000300010 >. Acesso em: 29 fev. 2012.

GARNERO, A. del V. et al. Parâmetros genéticos da taxa de maturação e do peso assintótico de fêmeas da raça Nelore. Arquivo Brasileiro de Medicina Veterinária e Zootecnia, v.57, p.652-662, 2005. Disponível em: <http://www.scielo.br/pdf/ abmvz/v57n5/26914.pdf>. Acesso em: 29 fev. 2012.

GIANOLA, D. et al. The Gibbs sampler in the animal model: a primer. In: FOULLEY, J.L.; MOLENAT, H. (Ed.). Séminaire modele animal. Departament de Genetique Animale, La Colle sur Loup, France: INRA, 1994. p.47-56.

JENKINS, T.G. et al. Evaluation of between and within breed variation in measures of weight-age relationships. Journal of Animal Science, v.69, p.3118-3128, 1991. Disponível em: <http://jas.fass.org/ content/69/8/3118.full.pdf+html>. Acesso em: 29 fev. 2012.

LOPES, F.B. et al. Ajustes de curvas de crescimento em bovinos Nelore da região Norte do Brasil. Revista Brasileira de Saúde e Produção Animal, v.12, p.607-617, 2011. Disponível em: <http:// revistas.ufba.br/index.php/rbspa/article/view/2145/1130>. Acesso em: 29 fev. 2012.

MALHADO, C.H.M. et al. Modelos não lineares utilizados para descrever o crescimento de bovinos da raça Nelore no estado da Bahia: 1. Efeito ambiental. Revista Brasileira de Saúde e Produção Animal, v.10, p.821-829, 2009. Disponível em: <http:// revistas.ufba.br/index.php/rbspa/article/view/1189/910>. Acesso em: 29 fev. 2012.

SANTORO, K.R. et al. Herdabilidades de parâmetros de curvas de crescimento não-lineares em zebuínos, no estado de Pernambuco. Revista Brasileira de Zootecnia, v.34, p.2280-2289, 2005. 
Disponível em: <http://www.scielo.br/pdf/rbz/v34n6s0/a14v3460. pdf>. Acesso em: 29 fev. 2012.

SAS INSTITUTE. Statistical analysis system: user's guide. Version 9.1. Cary, 2003. 5136p.

SILVA, N.A.M. et al. Modelo hierárquico bayesiano aplicado na avaliação genética de curvas de crescimento de bovinos de corte. Arquivo Brasileiro de Medicina Veterinária e Zootecnia, v.62, p.409-418, 2010. Disponível em: <http://www.scielo.br/pdf/ abmvz/v62n2/22.pdf>. Acesso em: 05 ago. 2012.

SILVA, N.A.M. et al. Seleção e classificação multivariada de modelos de crescimento não lineares para bovinos Nelore. Arquivo Brasileiro de Medicina Veterinária e Zootecnia, v.63, p.364-371, 2011. Disponível em: <http://www.scielo.br/pdf/ abmvz/v63n2/14.pdf>. Acesso em: 05 ago. 2012.

VAN KAAM, J.B.C.H.M. Gibanal. Analyzing program for Markov Chain Monte Carlo sequences version 2.3. Wageningen: Department of Animal Sciences, Wageningen Agricultural University, 1997. 4p. (Manual).

VAN TASSEL, C.P.; VAN VLECK, L.D. Multiple-trait gibbs sampler for animal models: flexible programs for bayesian and likelihood-based (co)variance component inference. Journal Animal Science, v.74, p.2586-2597, 1996. Disponível em: <http:// jas.fass.org/content/74/11/2586.full.pdf+html>. Acesso em: 29 fev. 2012 . 